\title{
Enhanced surveillance of hepatitis B infection in inner-western Sydney
}

\author{
Bradley L. Forssman ${ }^{\mathrm{A}, \mathrm{B}}$ and Leena Gupta ${ }^{\mathrm{A}}$ \\ ASydney South West Area Health Service Public Health Unit, \\ Sydney South West Area Health Service \\ ${ }^{\mathrm{B} C o r r e s p o n d i n g}$ author. Email: bforssman@optusnet.com.au
}

\begin{abstract}
Objectives: We developed an enhanced surveillance system for hepatitis B to improve the detection of newly acquired cases and to collect epidemiological data. Methods: The study was undertaken from February to June (inclusive) 2005 at the Sydney South West Area Health Service Eastern Zone Public Health Unit. A letter was sent to treating doctors on receipt of a notification, requesting additional information on cases. Cases identified by the treating doctors as newly acquired were followed up by telephone. Results: There were 295 notifications of hepatitis B in the period, of which three were newly acquired infections. Only one of these three cases was identified through enhanced surveillance. Information on ethnicity was obtained. Conclusions: This enhanced surveillance system is of limited value as an ongoing process. We recommend that it be undertaken periodically to monitor the epidemiology of the disease.
\end{abstract}

Hepatitis B is a viral infection that can cause chronic inflammation of the liver and may lead to cirrhosis and hepatocellular carcinoma. ${ }^{1}$ In Australia in 2005, 6793 unspecified hepatitis B notifications were reported to the National Notifiable Disease Surveillance System, with 235 of these reported as being incident (or newly acquired) cases. $^{2}$ In NSW, there were 54 newly acquired cases reported. ${ }^{2}$ Furthermore, it has been estimated that the prevalence of chronic hepatitis B infection in Australia ranges from $0.47-0.87 \%{ }^{3}$ Hepatitis $\mathrm{B}$ is preventable by vaccination, and further spread can be avoided through administration of immunoglobulin prophylaxis within 14 days of exposure, ${ }^{1}$ as well as through the provision of information about modes of transmission and infection control. In addition, new treatment modalities are proving to be effective. ${ }^{4}$ It would therefore be beneficial for cases of newly acquired hepatitis B to be detected in a timely manner to allow effective public health action to be instituted, as well as to facilitate early referral for management.

Identifying newly acquired cases of hepatitis B can be problematic, however, as laboratories usually only test and report results for positive surface antigen (HBsAg), which indicates unspecified infection with hepatitis $\mathrm{B}$, rather than IgM antibody to core antigen ( $\mathrm{HBcAb} \operatorname{IgM})$, which indicates newly acquired infection. In addition, clinical details or related results such as liver function tests are very rarely provided to the Public Health Unit (PHU). Identification of newly acquired cases is therefore highly dependent on the treating doctor notifying the PHU. While medical practitioners are required under the NSW Public Health Act 1991 to notify acute episodes of viral hepatitis, it is acknowledged that under-reporting is a significant issue., 5 In addition, laboratories provide limited demographic data (age, sex and postcode only), resulting in an incomplete picture of the epidemiology of hepatitis B.

A recent report of an enhanced surveillance system for hepatitis B in south-eastern Sydney stated that it was effective in increasing the detection of newly acquired cases by three-fold (from three to 10 cases) over a sixmonth period. ${ }^{7}$ However, this system required a reasonably high staff workload and relied on access to supplementary investigation results and clinical details. Consequently, it was unsuitable for implementation at our centre. An alternative enhanced surveillance system for hepatitis B infection was therefore developed, with the aims of improving both the detection of newly acquired cases and the collection of epidemiological data to inform targeted public health action. This article presents the results of this enhanced surveillance and discusses the implications of its implementation.

\section{Methods}

In NSW, hepatitis B infection is notifiable by laboratories and acute viral hepatitis is notifiable by medical practitioners and hospitals under the Public Health Act 1991. The case definition for newly acquired hepatitis B is provided in Box 1.8

Enhanced surveillance was undertaken from February to June (inclusive) 2005 at the Sydney South West Area Health Service (SSWAHS) Eastern Zone PHU, which services a large, ethnically diverse population of approximately 500000 in inner-western Sydney. ${ }^{9}$ After receipt of 
Box 1. Case definition for newly acquired hepatitis B infection ${ }^{8}$

- Detection of HBsAg in a patient shown to be negative within the last 24 months, or

- Detection of HBsAg and IgM to hepatitis B core antigen, in the absence of prior evidence of HBV infection, or

- Detection of HBV by nucleic acid testing, and IgM to hepatitis $B$ core antigen, in the absence of prior evidence of HBV infection.

Source: Notifiable Diseases Manual, NSW Department of Health 2004

a laboratory notification of HBsAg, a letter and two-page questionnaire were mailed to the medical practitioner who requested the test. Due to limited human resources, a reminder letter was not sent to treating doctors who did not respond. The letter outlined the reasons for undertaking this enhanced surveillance. The first page of the questionnaire requested demographic details of the case (gender, occupation, country of birth, language spoken at home, and Aboriginal and Torres Strait Islander status), and after providing the case definition of a newly acquired case of hepatitis $\mathrm{B}$, it requested the practitioner's opinion as to whether this case of hepatitis B was newly acquired. In addition, information regarding household or sexual contacts and the use of hepatitis B vaccine for these contacts was requested, as well as asking if the medical practitioner required further information regarding hepatitis $\mathrm{B}$, either for their patients or to assist with tertiary referrals.

If the case was considered newly acquired, the medical practitioner was asked to complete the second page of the questionnaire. This requested information regarding the presence and onset date of symptoms, and possible risk exposures. If further information was required for public health follow-up, it was obtained by telephoning the medical practitioner and the patient.

Data from returned questionnaires were entered into the NSW Health Notifiable Diseases Database and into a separate database created in Microsoft Excel 2000, and frequencies of selected characteristics were calculated.

In addition to the laboratory notifications of HBsAg, the PHU also received telephone notifications of acute hepatitis B directly from medical practitioners. The surveillance officers then obtained demographic and risk factor information regarding these cases from the medical practitioner and, if permission was given, directly from the patient. These cases were considered to be the 'baseline' number of notifications that would occur if the enhanced surveillance system was not in place.

The cost to the PHU of implementing this enhanced surveillance system was calculated by recording the amount of staff time taken for data entry, questionnaire and letter preparation and mail out, and telephone follow-up with treating doctors.

\section{Results}

\section{Enhanced surveillance}

There were 295 notifications of hepatitis B in the period February to July 2005 (average monthly notification rate of 9.9 per 100000 people). Median time taken to send questionnaires to treating doctors was 11 days from receipt of the laboratory notification (range 1-36 days). Three notifications were confirmed cases of newly acquired hepatitis B. Two of these were notified directly to the PHU by the treating medical practitioner, and the remaining case was detected as a result of the enhanced surveillance. There were eight additional cases that medical practitioners indicated on the questionnaire were newly acquired, but after follow-up by telephone it was determined that these cases did not meet the case definition for a newly acquired case. Out of the 293 laboratory notifications, 204 questionnaires were returned (response rate 69.6\%).

There were 104 cases (51\%) that had household contacts. Of these cases, it was indicated by medical practitioners that for 67 (64.4\%), hepatitis B vaccination had been instituted for their contacts.

Less than a quarter of medical practitioners requested further information regarding hepatitis $\mathrm{B}$, either for the information of their patients $(n=48,23.1 \%)$ or to assist in tertiary referral $(n=43,21.1 \%)$.

The additional staff time that this enhanced surveillance required was estimated to be four hours per week. If a public health nurse (5th year) were employed to perform these duties, the additional cost to the PHU would be approximately $\$ 85$ per week, or $\$ 4500$ per year. ${ }^{10}$ If, however, an existing public health surveillance officer were used to carry out this surveillance, this would likely be at the expense of an alternative communicable disease control activity.

\section{Demographics of cases}

The demographic characteristics of these cases are shown in Table 1 and country of birth data are shown in Figure 1. Cases were predominantly male (56\%) and aged 20-40 years. The majority of cases notified to the PHU spoke a language other than English at home (66\%) with 31\% speaking a Chinese dialect. Cases also tended to be born overseas, primarily in China (28\%) and Vietnam (21\%).

In all three cases of newly acquired hepatitis B, risk exposures were both unprotected sexual contact and intravenous drug use. These three people were all born in Australia, two were male and all were aged less than 30 years. 
Table 1. Demographic characteristics of people notified with hepatitis B, Sydney South West Area Health Service (Eastern Zone), NSW, February to July $2005(n=204)$

\begin{tabular}{llrr}
\hline Characteristic & & $\boldsymbol{n}$ & $\%$ \\
\hline Gender & Male & 114 & 55.9 \\
& Female & 89 & 43.6 \\
& Unknown & 1 & 0.5 \\
Age (years) & Median & 35 & \\
& Range & $7-90$ & \\
Language other than & & 134 & 65.7 \\
English spoken at home & & & \\
\hline
\end{tabular}

\section{Demographics of catchment population}

In the 2001 census, $50.6 \%$ of the PHU catchment population were male. The area is culturally diverse, with almost $40 \%$ of residents born overseas. The most common overseas countries of birth were China (4.2\%), the UK (3.5\%), Italy $(2.9 \%)$, Greece $(2.6 \%)$ and Lebanon $(2.3 \%){ }^{9}$

\section{Discussion}

This enhanced surveillance system was able to increase the detection of cases of newly acquired hepatitis B over a six-month period by one case. It also provided additional information regarding ethnicity that would otherwise not be available. In addition, it was helpful in obtaining some (albeit limited) data regarding the needs of medical practitioners with respect to information regarding hepatitis B, and the extent to which medical practitioners undertake appropriate management of contacts.

Our results differ from those of a recently reported enhanced surveillance system. ${ }^{7}$ This may be due to several reasons. We did not have access to the results of supple-

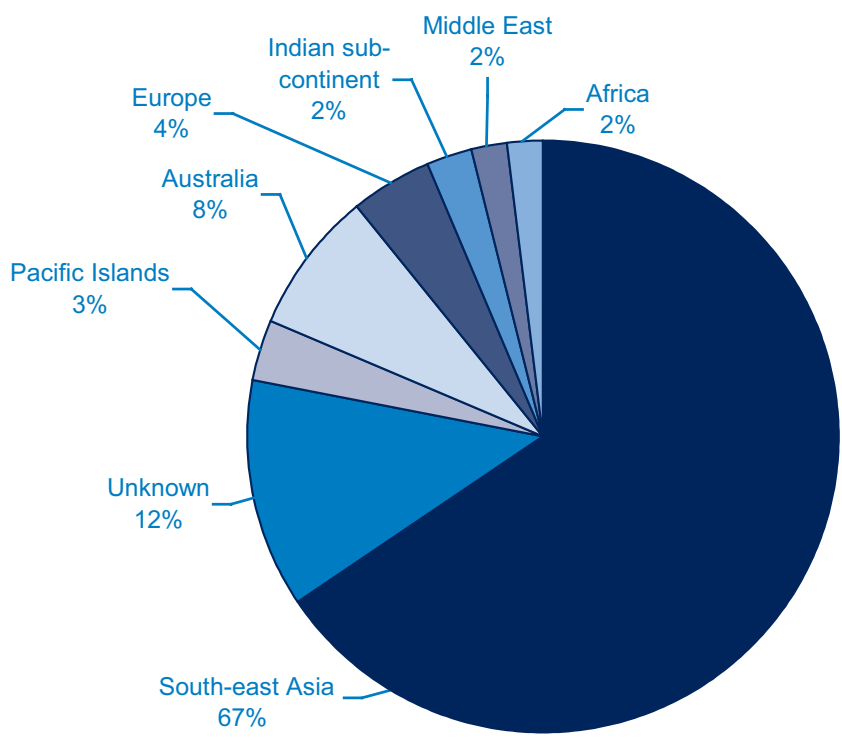

Figure 1. Country of birth of people notified with hepatitis B infection, Sydney South West Area Health Service (Eastern Zone), NSW, February to July $2005(n=204)$ mentary laboratory investigation or clinical details and were therefore unable to predict case classification before contacting treating doctors. This is unlikely to have made a difference to our results, demonstrated by the relatively large numbers of 'false positive' cases. It is possible that the treating doctors in our PHU catchment area do not under-report newly acquired cases of hepatitis B as is widely believed, and therefore enhanced surveillance for this reason is less important.

The inner-western region of Sydney has one of the highest notification rates of hepatitis B in NSW, which has been attributed to the large numbers of residents born in countries where hepatitis B is endemic. ${ }^{11}$ This is reflected in these results, where, compared with the demographic profile of the catchment population, a disproportionately high number of people notified with hepatitis B were born overseas, particularly in South-east Asia where hepatitis B carriage rates have been estimated to be up to $20 \% .^{12}$

Vaccination of household contacts of people with hepatitis B infection is known to reduce the risk of spread. ${ }^{1}$ Vaccination had been commenced in almost two-thirds of cases with household contacts, moderately lower than levels found in a previous study in northern Sydney $(88 \%),{ }^{13}$ but greater than studies in the United Kingdom $(38 \%)^{14}$ and the United States (55\%). ${ }^{15}$ Had there been more time between receipt of laboratory notification and mailing out of enhanced surveillance questionnaires, this vaccination rate may have been even higher. There could be other reasons for non-vaccination, however, such as previous vaccination, contraindication to vaccine, the failure of the contact to attend for vaccination, or the attendance of the contact to another provider for vaccination. This information was not collected by this enhanced surveillance, but it would be possible to do so in the future to enable a better understanding of reasons for the nonvaccination of contacts.

Despite the good response rate (which is likely to have been higher if additional human resources were available to send reminder letters to non-responding treating doctors), only one additional case of newly acquired hepatitis B was detected using this enhanced surveillance system, which did enable public health follow-up that would otherwise not have occurred. This was at the cost, however, of additional staff time spent in following up eight other cases that turned out to be 'false positives'. In addition, demographic information including ethnicity and Aboriginality could be collected on all cases of hepatitis $\mathrm{B}$, which assists in the understanding of the epidemiology of this disease, and could be used, in combination with other methods, to estimate the prevalence of hepatitis B. ${ }^{3}$ In the current climate of competing health funds, these benefits need to be weighed against the costs of such a surveillance system (including the time required by the 
treating doctors to complete the questionnaire). We would recommend that an option is to periodically undertake enhanced surveillance for a limited time, for example, six to 12 months every two to three years, to monitor the epidemiology of this disease. This would assist in filling the gap in data on the prevalence and incidence of this significant public health problem and contribute to informed public health practice.

\section{Acknowledgements}

We would like to thank Shamila Phillip for her assistance with data entry, and the medical practitioners who participated in this project.

\section{References}

1. Heymann DL, editor. Control of communicable diseases manual. 18th ed. Washington DC: American Public Health Association, 2004. pp. 253-261.

2. National Notifiable Disease Surveillance System, Population Health Division, Department of Health and Ageing. Available at http://www9.health.gov.au/cda/Source/Rpt_4_sel.cfm [Accessed 6 June 2007].

3. O'Sullivan BG, Gidding HF, Law M, Kaldor JM, Gilbert GL, Dore GJ. Estimates of chronic hepatitis B virus infection in Australia, 2000. Aust N Z J Public Health 2004; 28: 212-6.

4. Fung SK, Lok ASF. Update on viral hepatitis in 2004. Curr Opin Gastroentrol 2005; 21: 300-7. doi:10.1097/01.mog.0000158109.13722.36

5. Ferson MJ. Combined active-passive surveillance of acute hepatitis B. Commun Dis Intell 1995; 19: 258-9.

6. Alter MJ, Mares A, Hadler SC, Maynard JE. The effect of underreporting on the apparent incidence and epidemiology of acute viral hepatitis. Am J Epidemiol 1987; 125: 133-9.
7. Poulos RG, Ferson MJ. Enhanced surveillance of acute hepatitis B in south-eastern Sydney. Commun Dis Intell 2004; 28: 3 .

8. NSW Health. Hepatitis B response protocol for NSW Public Health Units, Notifiable Diseases Manual. Sydney: NSW Department of Health, 2004.

9. Wen LM, Manson A, Poulos R, Rissel CA. 2001 demographic profile of the Central Sydney Area Health Service. Camperdown: CSAHS Division of Population Health, 2003.

10. Office of Industrial Relations, NSW Department of Commerce. Available at http://www.industrialrelations. nsw.gov.au/awards/index.html [Accessed 6 June 2007].

11. Population Health Division. The health of the people of New South Wales - report of the Chief Health Officer. Sydney: NSW Department of Health. Available at http://www.health.nsw.gov.au/public-health/chorep/com/ com_hepbnot_ahs.htm [Accessed 6 June 2007].

12. Chen C, Wang L, Yu M. Epidemiology of hepatitis B virus infection in the Asia-Pacific region. J Gastro Hepatol. 2000; 15(Supp): E3-6. doi:10.1046/j.1440-1746.2000.02124.x

13. Staff MP, Angel PA. Vaccination among household contacts of chronic hepatitis B carriers by general practitioners. Aust Fam Physician 2002; 31: 491-3.

14. Richardson G, Evans MR, Westmoreland D. Hepatitis B immunisation of household contacts: retrospective study of vaccine coverage. $J$ Epidemiol Community Health 2001; 55: 934-5. doi:10.1136/jech.55.12.934

15. Weinberg MS, Gunn RA, Mast EE, Gresham L, Ginsberg M. Preventing transmission of hepatitis B virus from people with chronic infection. Am J Prev Med 2001; 20: 272-6. doi:10.1016/S0749-3797(01)00298-7 